\title{
The Large-Time Asymptotics of Some Wiener Integrals and the Interband Light Absorption Coefficient in the Deep Fluctuation Spectrum
}

\author{
W. Kirsch ${ }^{1}$ and L. A. Pastur ${ }^{2}$ \\ ${ }^{1}$ Institut für Mathematik, Ruhr-Universität Bochum, D-4630 Bochum, \\ Federal Republic of Germany and SFB 237: Unordnung und große Fluktuationen \\ ${ }^{2}$ Institute for Low Temperature Physics and Engeneering, \\ Academy of Sciences of the Ukrainian SSR, SU-310164 Kh'arkov, USSR
}

\begin{abstract}
In this paper we prove the existence of the interband-light-absorption coefficient and investigate its asymptotics for a number of models.
\end{abstract}

\section{Introduction}

The interband light absorption coefficient (ILAC) is an important characteristic of doped semiconductors. By using approximations widely accepted in semiconductor physics this quantity can be written as $[1,2]$ :

$$
\alpha(\lambda)=\frac{\alpha_{0}}{V \omega} \sum_{n, m} \delta\left(\lambda-\lambda_{n}^{+}-\lambda_{m}^{-}\right)\left|\int_{\Lambda} \psi_{n}^{+}(x) \overline{\psi_{m}^{-}(x)} d x\right|^{2},
$$

provided the temperature is sufficiently low and the Fermi energy lies in the gap between the valence and the conductance bands. In (1.1) the constant $\alpha_{0}$ is determined by fundamental physical constants and the band structure of the ideal (non-doped) semiconductor. $V$ denotes the volume of the semiconductor sample $\Lambda$. $\omega$ is the light frequency and $\lambda=-E_{g}+\omega$, where $E_{g}$ is the distance (gap) between the valence and the conduction band. $\lambda_{n}^{ \pm}$and $\psi_{n}^{ \pm}$are the eigenvalues and orthonormalized eigenfunctions respectively of the operators $H_{\Lambda}^{ \pm}$, given on $\Lambda$ by

$$
H_{\Lambda}^{ \pm}=-\Delta \pm q
$$

with appropriate (e.g. Dirichlet) boundary conditions on $\partial \Lambda$. These Hamiltonians describe the motions of electrons and holes in the conduction and valence bands under the influence of the random (impurity) potential $q(x)$. The energy levels $\lambda_{n}^{ \pm}$ are counted from the bottom of the respective band. ${ }^{1}$

It is known (see $[1,2])$ that the ILAC has several asymptotic regimes depending on the form of the random potential, the frequency interval, etc. In particular, suppose $q(x)$ is a homogeneous Gaussian random field with mean zero and cor-

1 For simplicity we consider only the case of equal effective masses of electrons and holes. This allows us to normalize the constants in front of the kinetic energy to 1 
relation function $b(x)=M\{q(0) q(x)\}^{2}$ which decays fast enough for $|x| \rightarrow \infty$. Then according to [2], for $-\lambda \gg b^{\frac{1}{2}}(0)$ we should have

$$
\ln \alpha(\lambda) \sim \ln n(\lambda) \text {. }
$$

Here the symbol $\sim$ denotes asymptotic equality and $n(\lambda)$ is the density of states of the operator $H_{A}^{ \pm}$. (It is clear that the density of states for $H_{A}^{ \pm}$agree due to the symmetry of the Gaussian distribution.) It is well known (see e.g. [1-3]) that for $-\lambda \gg b^{\frac{1}{2}}(0)$,

$$
\ln n(\lambda) \sim-\frac{\lambda^{2}}{2 b(0)} .
$$

In a recent paper [4] it is argued that if $b(x)=b(0) e^{-\gamma|x|}$ then

$$
\ln \alpha(\lambda) \sim \frac{1}{2} \ln n(\lambda) .
$$

In the present paper we give a rigorous proof of the asymptotic relation

$$
\ln \alpha(\lambda) \sim \frac{1}{2 \gamma_{1}} \ln n(\lambda),
$$

where $\gamma_{1}=1-\inf (b(x) / b(0))$. Due to ergodicity of the Gaussian field $q(x)$ a positive definite function $b(x)$ satisfies the inequality $|b(x)|<b(0)$, i.e. $1 \leqq \gamma<2$. Thus for general Gausian potential the asymptotic behaviour of the $\ln \alpha(\lambda)$ is not determined solely by the asymptotics of $\ln n(\lambda)$, like (1.3) or (1.5). This is the case only if $b(x)$ is nonnegative in which case we have (1.5). We also present arguments which show that for a certain class of random potentials it is natural to expect (1.5) when the random potential has symmetric distribution with noncompact support and the asymptotics (1.3) when the random potential is bounded from below or from above. In fact we prove for a certain class of random potentials that $\ln \alpha(\lambda) \sim \ln \left(n^{+} * n^{-}(\lambda)\right)$, where $n^{ \pm}$is the density of states for $H^{ \pm}$.

The paper is organized as follows. In the Sect. 2 we prove that the ILAC (1.1), more precisely the distribution function $A_{\Lambda}(\lambda)$ for which (1.1) is the density, with probability 1 has a nonrandom weak limit $A(\lambda)$ as $\Lambda \uparrow \mathbb{R}^{d}$ and obtain a representation for the Laplace transform $\tilde{A}(t)$ of $A(\lambda)$ in the form of a Wiener integral. Section 3 contains the proof of the asymptotic formulas for $A(\lambda)$ as $\lambda \rightarrow-\infty$ in the case of the Gaussian and the Poisson random potentials, which give in particular the rigorous variants of (1.5) and (1.3). This proof is based essentially on the mentioned representation. In Sect. 4 we investigate alloy type potentials. The last section concerns a brief discussion of our results and some related questions. In the appendix we prove that the construction of $A(\lambda)$ is independent of the boundary conditions chosen.

${ }^{2} M\{X\}$ denotes the expectation value of the random variable $X$ 


\section{Self-Averaging of the ILAC}

Denote by $\chi(\lambda)$ the characteristic function of the semiaxis $(0, \infty)$. Then the distribution function which corresponds to the density (1.1) is

where

$$
\begin{aligned}
A_{\Lambda}(\lambda) & =V^{-1} \sum_{m, n} \chi\left(\lambda-\lambda_{m}^{+}-\lambda_{n}^{-}\right) I_{m n}(\Lambda), \\
& =V^{-1} \operatorname{Sp} \int_{\mathbb{R}} E_{\Lambda}^{+}(\lambda-\mu) E_{\Lambda}^{-}(d \mu),
\end{aligned}
$$

$$
I_{m n}(\Lambda)=\left|\int_{\Lambda} \psi_{m}^{+}(x)\left(\psi_{n}^{-}(x)\right)^{*} d x\right|^{2}
$$

and $E_{\Lambda}^{ \pm}(\lambda)$ are the resolutions of identity of the operators $H_{\Lambda}^{ \pm}$defined by the Dirichlet problem in a region $\Lambda$ for the Schrödinger equations with the potentials $\pm q$ respectively acting in $L^{2}(\Lambda)$. We shall suppose in this paper that $\Lambda$ is a rectangular parallelepiped. Then the symbol $\Lambda / \mathbb{R}^{d}$ has an unambiguous meaning. ${ }^{3}$

Theorem 2.1. Let $q(x), x \in \mathbb{R}^{d}$, be a metrically transitive random field with continuous realizations and such that

$$
M\left\{e^{-t q(0)}\right\}<\infty, \quad t \in \mathbb{R} .
$$

Then there exists a non-random nondecreasing function $A(\lambda)$ such that

(i) with probability 1 (p. 1)

$$
\lim _{\Lambda \uparrow \mathbb{R}^{d}} A_{\Lambda}(\lambda)=A(\lambda)
$$

at all continuity points $\lambda$ of $A$.

(ii) If

$$
\tilde{A}(t)=\int_{\mathbb{R}} e^{-\lambda t} A(d \lambda)
$$

is the Laplace transform of $A(\lambda)$ then

$$
\tilde{A}(t)=\int M\left\{P^{+}(t, 0, x) P^{-}(t, x, 0)\right\} d x,
$$

where $P^{ \pm}(t, x, y), t \geqq 0, x, y \in \mathbb{R}^{d}$, are the kernels of the semigroups $e^{-t H^{ \pm}}$and $H^{ \pm}=-\Delta \pm q$ are random Schrödinger operators acting in $L^{2}\left(\mathbb{R}^{d}\right)$.

The proof of this theorem requires several auxiliary results. Denote by $\tilde{A}_{\Lambda}(t)$ the Laplace transform of (2.1):

$$
\tilde{A}_{\Lambda}(t)=V^{-1} \sum_{m, n} e^{-t\left(\lambda_{m}^{+}+\lambda_{n}^{-}\right)} I_{m n}=V^{-1} \mathrm{Sp} e^{-t H_{A}^{\dagger}} e^{-t H_{\bar{A}}} .
$$

If $P_{\Lambda}^{ \pm}(t, x, y), t \geqq 0, x, y \in \Lambda$ are the kernels of the semigroups $e^{-t H} \frac{t}{\Lambda}$, then

$$
\tilde{A}_{\Lambda}(t)=V^{-1} \int_{\Lambda} P_{\Lambda}^{+}(t, x, y) P_{\Lambda}^{-}(t, y, x) d x d y .
$$

3 Note that our results are valid for a much wider class of regions 
Applying the Cauchy inequality to the right-hand side of (2.7) we obtain that

where

$$
\tilde{A}_{\Lambda}(t) \leqq\left(\tilde{N}_{A}^{+}(2 t) \tilde{N}_{\Lambda}^{-}(2 t)\right)^{1 / 2},
$$

$$
\tilde{N}_{\Lambda}^{ \pm}(t)=V^{-1} \mathrm{Sp} e^{-t H_{A}}
$$

are the Laplace transforms of the prelimit densities of states $N_{\Lambda}^{ \pm}(\lambda)$ of $H_{A}^{ \pm}$:

$$
N_{A}^{ \pm}(\lambda)=V^{-1} \sum_{m} \chi\left(\lambda-\lambda_{m}^{ \pm}\right)
$$

According to the Feynman-Kac formula (see e.g. [6]) the kernels $P_{A}^{ \pm}(t, x, y)$ have the representations:

$$
P_{\Lambda}^{ \pm}(t, x, y)=p(t, x-y) E_{0, x}^{t, y}\left(e^{ \pm \int_{0}^{t} q(x(s))} \chi_{\Lambda}(x(\cdot))\right)
$$

where $p(t, x)=(4 \pi t)^{-\frac{d}{2}} e^{-\frac{x^{2}}{4 t}}, E_{0, x}^{t, y}$ denotes the Wiener expectation for $x(0)=x$ conditioned on $x(t)=y$ and

$$
\chi_{\Lambda}(x(\cdot))=\left\{\begin{array}{ll}
1 & \text { if } x(s) \in \Lambda, \text { all } 0 \leqq s \leqq t \\
0 & \text { otherwise }
\end{array} .\right.
$$

Lemma 2.2. If $\Lambda_{1} \subseteq \Lambda_{2}$ then

$$
0 \leqq P_{\Lambda_{1}}^{ \pm}(t, x, y) \leqq P_{A_{2}}^{ \pm}(t, x, y) .
$$

The proof of this lemma follows easily from the representation (2.10).

Lemma 2.3.

$$
\tilde{N}_{\Lambda}^{ \pm}(t) \leqq(4 \pi t)^{-\frac{d}{2}} V^{-1} \int_{\Lambda} e^{ \pm t q(x)} d x
$$

Proof. By using the Jensen inequality we have

$$
e^{ \pm \int_{0}^{t} q(x(x)+x)} \leqq t^{-1} \int_{0}^{t} e^{ \pm t q(x(s)+x)} d s
$$

This inequality (2.9), the Markov property of the Wiener process and (2.10) give $\mathrm{e}^{4}$

$$
\tilde{N}_{\Lambda}(t) \leqq V^{-1} \int_{\Lambda} p_{\Lambda}(t, x, x) e^{ \pm t q(x)} d x,
$$

where $p_{\Lambda}(t, x, y)$ is the kernel of the operator $e^{t \Delta_{\Lambda}}$ and $\Delta_{\Lambda}$ is the operator of the Dirichlet problem for the Laplacian. Applying to this kernel Lemma 2.2 with $q=0$, $\Lambda_{1}=\Lambda, \Lambda_{2}=R^{d}$ we arrive at $(2.12)$.

We can now prove Theorem 2.1. It follows from the Lemmas 2.2 and 2.3 that the quantity $V A_{\Lambda}(t)$ for fixed $t>0$ is a superadditive function of $\Lambda$, i.e. if $\Lambda=\Lambda_{1} \cup \Lambda_{2}$ and interior $\left(\Lambda_{1} \cap \Lambda_{2}\right)=\emptyset$ then

$$
V \tilde{A}_{\Lambda}(t) \geqq V_{1} \tilde{A}_{\Lambda_{1}}(t)+V_{2} \tilde{A}_{\Lambda_{2}}(t) .
$$

4 This inequality can be obtained also from Golden-Thompson inequality $\operatorname{Sp}\left\{e^{A+B}\right\} \leqq \operatorname{Sp} e^{A} e^{B}$ with $A=-\Delta_{A}^{D}, B= \pm q$. We use arguments based on (2.9) and (2.10) since similar ones will be used below in more complicated situations 
Thus according to the subadditive ergodic theorem [7] for fixed $t>0$ with $p$. 1 there exists the nonrandom limit

$$
\tilde{A}(t) \equiv \lim _{\Lambda \uparrow \mathbb{R}^{d}} \tilde{A}_{\Lambda}(t)
$$

Now to prove (2.4) we use a simple generalization [8] of the well known continuity theorem for the Laplace transform of nondecreasing functions. The conditions required for this theorem are satisfied because of (2.3) and (2.12).

To prove (2.5) we take into account that according to the subadditive ergodic theorem

$$
\tilde{A}(t)=\lim _{\Lambda \uparrow \mathbb{R}^{d}} M\left\{\tilde{A}_{\Lambda}(t)\right\}
$$

Thus, since due to the metrical transitivity of the potential $M\left\{P^{+}(t, x, y) P^{-}(t, y, x)\right\}$ depends only on the difference $x-y$ and for any $\phi(x) \in L_{1}\left(\mathbb{R}^{d}\right)$,

$$
\lim _{\Lambda \uparrow \mathbb{R}^{d}} V^{-1} \int_{\Lambda^{2}} \phi(x-y) d x d y=\int \phi(x) d x
$$

we have only to prove that for any fixed $t>0$

$$
\lim _{\Lambda \perp \mathbb{R}^{d}} M\left\{V^{-1} \operatorname{Sp}\left(P^{+} P^{-}-P_{\Lambda}^{+} P_{\Lambda}^{-}\right)\right\}=0,
$$

where $P^{ \pm}$and $P_{A}^{ \pm}$are the integral operators in $L^{2}(\Lambda)$ defined by the kernels $P^{ \pm}(t, x, y)$ and $P_{A}^{ \pm}(t, x, y)$ for fixed $t>0$. But the expression under the lim sign in the left-hand side of (2.16) can be written as $S_{1}+S_{2}$, where

$$
\begin{aligned}
& S_{1}=M\left\{V^{-1} \operatorname{Sp} P_{A}^{+}\left(P^{-}-P_{\Lambda}^{-}\right)\right\}, \\
& S_{2}=M\left\{V^{-1} \operatorname{Sp} P^{-}\left(P^{+}-P_{A}^{+}\right) .\right.
\end{aligned}
$$

By using (2.11) and the Cauchy inequality we obtain that

$$
0 \leqq S_{1} \leqq\left(M\left\{V^{-1} \operatorname{Sp}\left(P_{A}^{+}\right)^{2}\right\} M\left\{V^{-1} \operatorname{Sp}\left(P^{-}-P_{A}^{-}\right)^{2}\right\}\right)^{1 / 2}
$$

It follows from the semigroup property of the $P_{A},(2.11)$ and (2.12) that the first factor in the right-hand side of (2.18) admits the estimate

$$
(8 \pi t)^{-d / 2} M\left\{e^{-2 t q(0)}\right\}<\infty .
$$

Furthermore, writing down the Wiener integral representation for the difference $P^{-}-P_{\Lambda}^{-}$which follows from (2.10) and applying to it the Cauchy inequality we obtain that

$$
\left(P^{-}-P_{A}^{-}\right)^{2}(t, x, y) \leqq\left(p-p_{A}\right)(t, x, y) p(t, x-y) E_{0, x}^{t, y}\left(e^{2 \int_{0}^{t} q(x(s)) d s}\right) .
$$

The mathematical expectation of the right-hand side of this inequality in view of (2.13) has the estimate

$$
V^{-1} \operatorname{Sp}\left\{p\left(p_{\Lambda}-p\right)\right\} M\left\{e^{2 t q(0)}\right\}
$$

which shows that $\lim _{\Lambda \uparrow \mathbb{R}^{d}} M\left\{S_{1}\right\}=0$ if $\lim _{\Lambda \uparrow \mathbb{R}^{d}} V^{-1} \operatorname{Sp}\left\{p\left(p_{\Lambda}-p\right)\right\}=0$. But since the functions $p(t, x, y)$ and $p_{\Lambda}(t, x, y)$ are rather simply connected [9] ( $p_{\Lambda}$ is obtained from $p$ by alternating reflecting procedure) the last limit relation can be proved by direct calculations. In a similar way we establish that $\lim _{\Lambda \uparrow \mathbb{R}^{d}} S_{2}=0$. 
Corollary 2.1. The Laplace transform $\tilde{A}(t)$ of $A$ is given by:

$$
\tilde{A}(t)=\int p^{2}(t, x) E_{0,0}^{t, x} \times E_{0,0}^{t, x} \times M\left(e^{-\int_{0}^{t} q(x(s))-q(x-y(s)) d s}\right) d x .
$$

Here $E_{0, x}^{t, y}$ is expectation over the Wiener measure conditioned by $x(0)=x$ and $x(t)$ $=y$. The first expectation is over $x(\cdot)$, the second one over $y(\cdot)$.

This corollary follows from (2.5) and (2.10). Finally, we prove an estimate on $\tilde{A}(t)$ which we will use later on.

Proposition 2.1. Under the assumptions of Theorem 2.1 the Laplace transform $\tilde{A}(t)$ of $A(\lambda)$ satisfies:

$$
\tilde{A}(t) \leqq \int p(t, x)^{2} M\left\{e^{-t(q(0)-q(x))}\right\} d x .
$$

Remark. The analogous result on the IDS:

$$
\tilde{N}(t) \leqq p(t, 0) M\left\{e^{t q(0)}\right\}
$$

is well known (see e.g. [8]). It follows, for example, from (2.12).

Proof.

$$
\begin{aligned}
\tilde{A}(t)= & \int p(t, x)^{2} M \times E_{0,0}^{t, x}(x(\cdot)) \times E_{0,0}^{t, x}(y(\cdot)) \\
& \left(e^{-\int_{0}^{t}(q(x(s))-q(x-y(s)) d s}\right) d x \\
\leqq & \int p(t, x)^{2} \frac{1}{t} \int_{0}^{t} d s M \times E_{0,0}^{t, x}(x(\cdot)) \times E_{0,0}^{t, x}(y(\cdot)) \\
& \left(e^{-t(q(x(s))-q(x-y(s))}\right) d x
\end{aligned}
$$

by application of Jensen's inequality,

$$
\begin{aligned}
= & \frac{1}{t} \int_{0}^{t} d s \int_{\mathbb{R}^{d}} M \int_{\mathbb{R}^{d}} \int_{\mathbb{R}^{d}}(p(s, y) p(t-s, y-x) p(s, x-z) \\
& \left.p(t-s, z) e^{-t(q(y)-q(z))}\right) d z d y d x \\
= & \frac{1}{t} \int_{0}^{t} d s M\left\{\iiint p(s, y) p(t-s, y-x) p(s, z)\right. \\
& \left.p(t-s, z+x) e^{-t q(y)-q(z+x)}\right\} d z d y d x \\
= & \frac{1}{t} \int_{0}^{t} d s M\left\{\iiint p(s, y) p(t-s, x-z) p(s, z)\right. \\
& \left.p(t-s, x+y) e^{-t(q(y)-q(x+y))}\right\} d x d z d y \\
= & \frac{1}{t} \int_{0}^{t} d s \iiint p(s, y) p(t-s, y+x) p(s, z) \\
& p(t-s, z-x) M\left\{e^{-t(q(y)-q(x+y))}\right\} d z d y d x \\
= & \int p(t, x)^{2} M\left\{e^{-t(q(0)-q(x))}\right\} d x .
\end{aligned}
$$

In the last step we used the stationarity of $q$. 
Remark. One could, of course, define (2.1) by imposing Neumann boundary conditions rather than Dirichlet ones. We prove in the Appendix that this procedure leads to the same limit $A(\lambda)$. This is therefore also true for any boundary conditions between Dirichlet and Neumann, e.g. for periodic boundary conditions.

\section{Asymptotic Behaviour of the ILAC for the Gaussian and the Poisson Potentials}

In this section we prove the following results on the asymptotic behaviour of the ILAC:

Theorem 3.1. Let $q(x)$ be a metrically transitive Gaussian random field with continuous correlation function $b(x)$.

Then

$$
\ln A(\lambda)=-\frac{\lambda^{2}}{4}\left[b(0)-\inf _{x \in \mathbb{R}^{d}} b(x)\right]^{-1}(1+o(1)) \quad \text { as } \lambda \rightarrow-\infty .
$$

Let us define now the Poisson random potential. Denote by $\mu(d x)$ the Poisson random measure $\mu(d x) \in \mathbb{R}^{d}$. This is the pure atomic measure such that the random variables $\mu\left(\Delta_{1}\right)$ and $\mu\left(\Delta_{2}\right)$ are independent for any Borel sets in $\mathbb{R}^{d}$, if $\Delta_{1} \cap \Delta_{2}$ has empty interior, and the random variable $\mu(\Delta)$ takes on nonnegative integer values $n=0,1 \ldots$ with probabilities $e^{-c|\Delta|} \frac{(c|\Delta|)^{n}}{n !}$, where $c>0$ (density) and $|\Delta|$ is the Lebesgue measure of the Borel set $\Delta$. For a smooth function $f(x)$ with compact support the integral

$$
q(x) \equiv \int f(x-y) \mu(d y)
$$

defines with probability 1 a metrically transitive random field, which we will call the Poisson random potential. This potential is more complex and general than the Gaussian one. In particular, if $f(x)$ in (3.2) has the form $f(x)=g u(x)$ and $c \rightarrow \infty$, $g \rightarrow 0, g^{2} c=1$ then all joint probability distributions of the random variables $v\left(x_{1}\right) \ldots v\left(x_{n}\right), v\left(x_{1}\right)=q\left(x_{1}-M(q(x))\right)$, tend to the respective joint distributions of the Gaussian potential with zero mean value and the correlation function of the form $b(x)=\int u(x-y) u(y) d y$.

Theorem 3.2. Suppose that the function $f(x)$ in (3.2) has a unique negative and nondegenerate minimum at $x=0$ and a unique nonnegative and nondegenerate maximum at $x=x_{0}$. Then

$$
\ln A(\lambda)=-\frac{\lambda}{f(0)-f\left(x_{0}\right)} \ln \frac{\lambda}{f(0)-f\left(x_{0}\right)}(1+o(1)), \quad \text { as } \lambda \rightarrow \infty .
$$

As in the case of the integrated density of states (see e.g. [8]) we can obtain the above asymptotic formulas from a corresponding formula for the Laplace transform $\tilde{A}(t)$ of $A(\lambda)$ by means of the Minlos-Povsner Tauberian theorem. This is easily done in the same way as in [8] once we known the asymptotic behaviour of $\tilde{A}(t) \rightarrow+\infty$. 
Proposition 3.1. Under the assumption of Theorem 3.1 we have

$$
\ln \tilde{A}(t)=\gamma_{1} b(0) t^{2}(1+o(1)) \text { as } t \rightarrow \infty,
$$

where $\gamma_{1}=1-\inf _{x \in \mathbb{R}^{d}} b(x) / b(0)$.

Proof. As in the case of the integrated density of states we obtain (3.3) as a result of upper and lower bounds which agree asymptotically for large $t$. We use heavily the Wiener integral representation of $\tilde{A}(t)$ given in (2.19).

Upper Bond. By Proposition 2.1 we have

$$
\begin{aligned}
\tilde{A}(t) & \leqq \int p(t, x)^{2} M\left\{e^{-t(q(0)-q(x))}\right\} d x \\
& =\int p(t, x)^{2} e^{\frac{1}{2} t^{2} M\left([q(0)-q(x)]^{2}\right)} d x
\end{aligned}
$$

since $q$ is Gaussian and:

$$
=e^{t^{2} M(q(0))} \int p(t, x)^{2} e^{-t^{2} M(q(0) q(x))} d x
$$

by stationarity of $q(x)$, thus:

$$
\leqq C \exp \left(t^{2}\left[b(0)-\inf _{x \in \mathbb{R}^{d}} b(x)\right]\right)
$$

for $t$ large enough.

Lower Bound. Set $\Omega_{1}=\{x(\cdot)|| x(s) \mid \leqq \eta$ for all $0 \leqq s \leqq t-\delta\}, \Omega_{2}=\{y(\cdot)|y(s)| \leqq \eta$ for all $0 \leqq s \leqq t-\delta\}$, where $\delta$ and $\eta$ will be determined later and let $x_{0}$ be a point such that inf $b(x)=b\left(x_{0}\right)$ (if $b(x) \geqq 0$ then any point $x_{0},\left|x_{0}\right| \geqq R$ for $R$ large enough, may $x \in \mathbb{R}^{d}$

play this role). Then

$$
\begin{aligned}
\tilde{A}(t) \geqq & \int_{\left|x-x_{0}\right| \leqq \eta} p(t, x)^{2} \\
& \int_{\Omega_{1}} \int_{\Omega_{2}} M\left\{e^{-\int_{0}^{t} q(x(s))-q(x-y(s)) d s}\right\} d P_{0,0}^{t, x} d P_{0,0}^{t, x} d x .
\end{aligned}
$$

The random variable in the exponent is Gaussian, hence

$$
\begin{aligned}
& M\left(e^{-\int_{0}^{t}(q(x(s))-q(x-y(s)) d s}\right) \\
& \quad=e^{\frac{1}{2} \int_{0}^{t} d s^{\prime} \int_{0}^{t} d s\left(b\left(x(s)-x\left(s^{\prime}\right)\right)+b\left(y\left(s^{\prime}\right)-y(s)\right)-2 b\left(x(s)+y\left(s^{\prime}\right)-x\right)\right)} .
\end{aligned}
$$

Take $\varepsilon>0$ and take $\eta$ so small that $\left|b\left(z_{1}\right)-b(0)\right|<\varepsilon / 2,\left|b\left(z_{2}+x_{0}\right)-\left(b\left(x_{0}\right)\right)\right| \leqq \varepsilon / 2$ for $\left|z_{1}\right|,\left|z_{2}\right| \leqq \eta$. Then for $x(\cdot) \in \Omega_{1}, y(\cdot) \in \Omega_{2}$ this expectation is not less than

$$
\exp \left\{(t-\delta)^{2}\left[b(0)-b\left(x_{0}\right)-\varepsilon\right]-K \delta t\right\}
$$

for a suitable constant $K$. Hence

$$
\begin{aligned}
\tilde{A}(t) \geqq & C \exp \left\{(t-\delta)^{2}\left[b(0)-b\left(x_{0}\right)-\varepsilon\right]-K \delta t\right\} \\
& \int_{\left|x_{1}\right|,\left|x_{2}\right|,\left|x_{3}\right| \leqq \eta} p_{\eta}\left(t-\delta, 0, x_{1}\right) p\left(\delta, x_{0}-x_{1}+x_{2}\right) \\
& p_{\eta}\left(t-\delta, x_{2}, x_{3}\right) p\left(\delta, x_{0}+x_{3}\right) d x_{1} d x_{2} d x_{3}
\end{aligned}
$$


where $p_{\eta}(s, x-y)$ is the probability that the Wiener trajectory $x(\tau), x(0)=x$, $x(s)=y$, satisfies the inequality $|x(\tau)| \leqq \eta, 0 \leqq \tau \leqq s$. This probability is the solution of the heat equation for which $p_{\eta}(0, x, y)=\delta(x-y),|x|,|y| \leqq \eta,\left.p_{\eta}(\tau, x, y)\right|_{|x|=\eta}$ $=\left.p_{\eta}(\tau, x, y)\right|_{|y|=\eta}=0,0 \leqq \tau \leqq s$. Based on this fact, it is easy to show that the integral on the right-hand side of (3.4) has a lower bound $C_{1} e^{-C_{2} t / \eta^{2}}$ for some constants $C_{1}$ and $C_{2}$. As a result

thus

$$
\ln \tilde{A}(t) \geqq(t-\delta)^{2}\left(b(0)-\varepsilon-b\left(x_{0}\right)\right)+\left(\frac{C_{2}}{\delta^{2}}+K \delta\right) t+O(1),
$$

$$
\left.\lim _{t \rightarrow \infty} \frac{\ln \tilde{A}(t)}{t^{2}} \geqq\left(b(0)-\varepsilon-b\left(x_{0}\right)\right)\right),
$$

and sending $\varepsilon \rightarrow 0$ and $R \rightarrow \infty$ we obtain the desired bound.

Remark. For $b(x) \geqq 0$ the result of the proposition may be interpreted as a kind of asymptotic factorization of $\tilde{A}(t)$ into $\tilde{N}^{+}(t) \tilde{N}^{-}(t)$ since it is known that $\tilde{N}^{ \pm}$behave like $\exp \left\{t^{2} \frac{b(0)}{2}(1+o(1))\right\}$ (see [8]). This can be interpreted as the more or less independent behaviour of the electrons and holes in such a Gaussian potential: electrons and holes dig their own wells. In our proof this is reflected in the form of trajectories which gives the main contribution to the Wiener integral representing $\tilde{A}(t)$. Recall that in the case of the density of states the main contribution to the respective representation of its Laplace transform is given by the trajectories which remain close to the origin all the time. It is easy to see that these paths are close to the classical one because the classical path $x_{\mathrm{cl}}(s)$ is the one which gives maximum to the "action" functional

$$
-\int_{0}^{t} \dot{x}^{2}(s) d s+\frac{1}{2} \int_{0}^{t} \int_{0}^{t} d s d s^{\prime} b^{\prime}\left(x(s)-x\left(s^{\prime}\right)\right)
$$

on the set $\{x(0)=x(t)=0\}$, so $x_{\mathrm{cl}}(s) \equiv 0$. The action functional to be considered in the case of the ILAC reads

$$
\begin{aligned}
-\int_{0}^{t}\left[\dot{x}^{2}(s)\right. & \left.+\dot{y}^{2}(s)\right] d s+\frac{1}{2} \int_{0}^{t} \int_{0}^{t} d s d s^{\prime}\left[b^{\prime}\left(x(s)-x\left(s^{\prime}\right)\right)\right. \\
& \left.+b^{\prime}\left(y(s)-x\left(s^{\prime}\right)\right)-2 b^{\prime}\left(x(s)-y\left(s^{\prime}\right)\right)\right] .
\end{aligned}
$$

In this case the classical trajectoties $x_{\mathrm{cl}}(s)$ and $y_{\mathrm{cl}}(s)$ are given by

$$
x_{\mathrm{cl}}(s)=s x \text { and } y_{\mathrm{cl}}(s)=(1-s / t) x
$$

respectively. It is easy to see that they give the minimum rather than the maximum of our action. The maximum, however, is given by the paths that remain close to zero (respectively $x_{0}$ ) up to time $t$ when they jump to $x_{0}$ (respectively 0 ).

Proposition 3.2. Under the assumption of Theorem 3.2 we have

$$
\ln \tilde{A}(t)=c\left((2 \pi t)^{d} D\right)^{-\frac{1}{2}} e^{t \gamma f(0)}(1+o(1)), \quad \text { as } t \rightarrow+\infty,
$$

where $v=1-f\left(x_{0}\right) / f(0)=1-\inf f(x) / \sup f(x)$ and $D$ is the Hessian of the function $f(y)-f\left(y-x_{0}\right)$ at $\left.y=0\right)$. 
Proof. We will follow the scheme of the proof of Proposition 3.1. We need as in the case of the Gaussian potential the explicit form of the generating functional

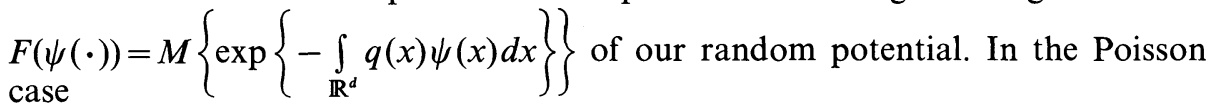

$$
F(\psi(\cdot))=\exp \left\{c \int d x\left(e^{-\int f(x-y) \psi(y) d y}-1\right)\right\} .
$$

Upper Bound. Proposition 2.5 and (3.6) for $\psi(x)=\delta(x)-\delta(x-y)$ give the inequality

$$
\tilde{A}(t) \leqq \int p^{2}(t, x) \exp \left\{c \int\left(e^{t f(y)-t f(y-x)}-1\right) d y\right\} .
$$

Take positive and small number $\eta_{1}$ and $\eta, \eta_{1}>\eta$. Then it is easy to show that the right-hand side $I(t)$ of (3.7) admits the estimate

where

$$
I(t) \leqq C\left(\eta_{1}\right) t^{-d} \sup _{\left|x-x_{0}\right| \leqq \eta_{1}} \exp \left\{c I_{1}(t, x)+I_{2}\right\},
$$

$$
\begin{gathered}
I_{1}(t, x)=\int_{|y| \leqq \eta} e^{t f(y-x)-t f(y)} d y, \\
I_{2}=O\left(t \cdot \operatorname { M a x } \left\{\exp \left\{t\left[f\left(x_{0}\right)-\inf _{|y| \geqq \eta} f(\eta)\right]\right\},\right.\right. \\
\left.\exp \left\{t\left[\sup _{|y| \geqq \eta_{1}-\eta} f\left(x_{0}+y\right)-f(0)\right]\right\}\right\} .
\end{gathered}
$$

By using standards arguments of the Laplace method one can find that

$$
I(t, x)=\left[(2 \pi t)^{d} D_{x}\right]^{-1 / 2} \exp \left\{t \sup _{|y| \leqq \eta}[f(y-x)-f(y)]\right\}(1+o(1)), \quad t \rightarrow \infty,
$$

where $D_{x}$ is the Hessian of the function $f(\cdot)-f(\cdot-x)$ at its maximum. It is important that due to the smoothness of $f(x)$ and smallness of $\eta_{1}$ and $\eta$ this asymptotic formula holds uniformly in $x,\left|x-x_{0}\right| \leqq \eta$. Thus inserting (3.11) in (3.8) we can replace with small error $o(1)\left(\right.$ as $\left.\eta_{1}, \eta \rightarrow 0\right), D_{x}$ by $D$ and take into account that

$$
\sup _{\left|x-x_{0}\right| \leqq \eta_{1}} \sup _{|y|<\eta}[f(y-x)-f(y)]=f\left(x_{0}\right)-f(0) .
$$

Since by our conditions, inf $f(y)<f(0),|y|<\eta, \forall \eta>0, \sup _{\left|x-x_{0}\right|>\eta} f(x)<f\left(x_{0}\right)$, we obtain the right-hand side of (3.5) as an asymptotic upper bound for $\ln \tilde{A}(t)$ as $t \rightarrow+\infty$.

Lower Bound. Corollary 2.1 and (3.6) with

$$
\psi(y)=\int_{0}^{t}[\delta(x(s)-y)-\delta(x-y-y(s))] d s
$$

give that in the Poisson case

$$
\tilde{A}(t)=\int p^{2}(x, t) E_{(0,0)}^{(t, x)}\left(\exp \left\{c \int\left(e^{\frac{f}{\int} f(y-x(s)) d s} e^{\int_{0}^{t} f(y-x+y(s)) d s}-1 d y\right)\right\}\right) d x .
$$

Now as in Proposition 3.1 we restrict the Wiener integrals to the set $\Omega_{1}$ and $\Omega_{2}$ respectively for the trajectories $x(\cdot)$ and $y(\cdot)$. After that we split the $y$-integral in the 
exponent of (3.12) on the integrals over the domains $|y| \geqq \eta_{1},|y| \leqq \eta_{2}, \eta_{1}>\eta$. The first integral does not exceed $t\|u\| \exp \left(t\left[f\left(x_{0}\right)-\inf _{|y| \geqq \eta_{1}-\eta} f(y)\right]\right.$. The second integral on the set $\Omega_{1} \times \Omega_{2}$ admits the following asymptotic estimate:

$$
\begin{aligned}
e^{O(t \eta)} & \int_{|y| \leqq \eta_{1}} e^{t\left[f\left(y-x_{0}\right)-f(y)\right]} d y-O\left(\eta_{1}^{d}\right) \\
& =e^{O(t \eta)}\left((2 \pi t)^{d} D\right)^{-\frac{1}{2}} e^{t\left[f\left(x_{0}\right)-f(0)\right]}\left(1+o(1)+o\left(\eta_{1}^{d}\right)\right), \quad t \rightarrow+\infty
\end{aligned}
$$

Let us choose now $\eta=O\left(t^{-1-a}\right), a>0$. Then $O(t \eta)=O\left(t^{-a}\right)=o(1), t \rightarrow+\infty$, and $e^{o(t \eta)}=1+o(1), t \rightarrow \infty$. Since the Wiener measure of the set $\Omega_{1} \times \Omega_{2}$ has the lower estimate $C_{1} e^{-c_{2} t / \eta^{2}}$ (see Proposition 3.1) the contribution of this measure to the lower bound of $\ln \tilde{A}(t)$ is of the order $O\left(t^{3+2 a}\right)$. This contribution is negligible in comparison with the exponentially growing contribution (3.13) due to the Poisson potential. Thus the first term of the right-hand side of (3.13) is the leading term of the lower bound of $\ln \tilde{A}(t)$, i.e. this bound asymptotically coincides with the righthand side of (3.5). The proof is complete.

Remark. The conditions which we imposed on the function $f(x)$ are not optimal. We chose them to demonstrate with minimal technicalities the difference in the asymptotic behaviour of the ILAC and the IDS (or their Laplace transforms). We see that the latter contains only the value $f(x)$ at its negative minimum while the former contains also the value $f(x)$ at its nonnegative maximum.

\section{IDS and ILAC for Alloy-Type Potentials}

In this section we consider an easy case of an alloy-type potential

$$
q(x)=\sum_{i \in \mathbb{Z}^{d}} \xi_{i} f(x-i)
$$

with $\xi_{i}$ independent, identically distributed and $f$ a $C^{2}$-function of support inside the unit cell $C_{0}=\left\{\left(x_{1}, \ldots, x_{d}\right) \mid-1 / 2<x_{i}<1 / 2, i=1, \ldots, d\right\}$. Furthermore, we assume that $f$ has a unique nondegenerate maximum at $x=x_{+}$and a unique nondegenerate minimum at $x=x_{-}$. Our method applies also in the case of a unique nondegenerate extremum if $f$ has definite sign. More general alloy-type potentials will be considered elsewhere. The IDS of alloy-type potentials is known to exist under very mild assumptions (see e.g. [10]). In fact, the so-called suspension trick allows us to carry over results of Sect. 2 without further work (see [11]). If

$$
M\left(e^{-t \xi_{0}}\right)<\infty
$$

for all $t \in \mathbb{R}$ then the IDS $N(\lambda)$ and the ILAC $A(\lambda)$ exist and their Laplace transforms are given by

and

$$
\tilde{N}(t)=E_{0,0}^{t, 0} \int_{C_{0}} d u M\left(e^{-\int_{0}^{t} q(x(s)+u) d s}\right)
$$

$$
\begin{aligned}
\tilde{A}(t)= & \int_{\mathbb{R}^{d}} p^{2}(t, x) E_{0,0}^{t, x}(d x(\cdot)) E_{0, x}^{t, 0}(d y(\cdot)) \\
& \int_{C_{0}} d u M\left(e^{-\int_{0}^{t}(q(x(s)+u)-q(y(s)+u)) d s}\right)
\end{aligned}
$$


respectively. The additional integration over $d u$ comes from the fact that (4.1) is stationary merely with respect to lattice translations. In the sequel we always assume (4.2).

First we give the asymptotic behaviour of $\tilde{N}(t)$ for $t \rightarrow \infty$ if the random variable $\xi_{0}$ can assume arbitrarily negative values. As usual results on $N(\lambda)$ can be deduced from this by means of a Tauberian theorem.

Denote by $P_{0}$ the distribution of $\xi_{0}$ and by $F$ the corresponding distribution function, i.e. $F(\lambda)=P_{0}((-\infty, \lambda))$. We will write $\tilde{F}$ for its Laplace transform.

Theorem 4.1. If supp $P_{0}$ is unbounded then

Proof.

$$
\ln \tilde{N}(t) \sim \ln \left(\tilde{F}\left(t f\left(x_{+}\right)\right)+\tilde{F}\left(t f\left(x_{-}\right)\right)\right) \text {as } t \rightarrow \infty \text {. }
$$

\section{Upper Bound.}

$$
\begin{aligned}
\tilde{N}(t) & \leqq p(t, 0) \int_{C_{0}} d u M\left(e^{-t \xi_{0} f(u)}\right) \\
& =p(t, 0) \int_{C_{0}} d u\left\{M_{-}\left(e^{-t \xi_{0} f(x+)}\right)+M_{+}\left(e^{t \xi_{0} f\left(x_{-}\right)}\right)\right\},
\end{aligned}
$$

where $M_{ \pm}$denotes expectation over the sets

$$
\begin{aligned}
\Omega_{+} & =\left\{\xi_{0} \geqq O\right\} \text { and } \Omega_{-}=\left\{\xi_{0}<0\right\} \\
& \leqq p(t, 0)\left\{\tilde{F}\left(t f\left(x_{+}\right)\right)+\tilde{F}\left(t f\left(x_{-}\right)\right)\right\}
\end{aligned}
$$

\section{Lower Bound.}

$$
\tilde{N}(t)=E_{0,0}^{t, 0} \int_{C_{0}} d u M\left(e^{-\int_{0}^{t} q(x(s)+u) d s}\right) .
$$

We restrict the integration over $x(s)$ to those path which stay close to 0 , i.e. to the set $\Omega_{0}=\{x(\cdot) \mid x(s) \leqq \delta$ for all $s \leqq t\}$. By taking $\delta$ small enough we obtain

$$
\tilde{N}(t) \geqq \int_{\Omega_{0}} d \mathbb{P}_{0,0}^{t, 0} \times M \int_{C_{0}} d u\left(e^{-\xi_{0} \int_{0}^{t} f(x(s)+u) d s}\right) .
$$

For any $\varepsilon>0$ we may take $\delta$ so small that on $\Omega_{0}|f(x(s)+u)-f(u)|<\varepsilon$, hence

$$
\tilde{N}(t) \geqq \int_{\Omega_{0}} d \mathbb{P}_{0,0}^{t, 0}\left\{M_{+} \int_{C_{0}} d u\left(e^{-t \xi_{0}(f(u)+\varepsilon)}\right) M_{-} \int_{C_{0}} d u\left(e^{-t \xi_{0}(f(u)-\varepsilon)}\right)\right\} .
$$

By Laplace's method we get (for $t$ large)

$$
\geqq C \mathbb{P}_{0,0}^{t, 0}\left(\Omega_{0}\right) \cdot t^{-d / 2}\left\{M_{+}\left(e^{-t \xi_{0}\left(f\left(x_{+}\right)+2 \varepsilon\right)}\right) M_{-}\left(e^{-t \xi_{0}\left(f\left(x_{-}\right)-2 \varepsilon\right)}\right)\right\} .
$$

Since $\mathbb{P}_{0,0}^{t, 0}\left(\Omega_{0}\right) \geqq C_{1} e^{-c_{2} \frac{t}{\delta^{2}}}$ we conclude again that

$$
\lim _{t \rightarrow \infty} \frac{\ln \tilde{N}(t)}{\ln \left\{\tilde{F}\left(t f\left(x_{-}\right)\right)+\widetilde{F}\left(t f\left(x_{+}\right)\right)\right\}} \geqq 1 .
$$

Theorem 4.2. If $\sup P_{0}$ is unbounded then

as $t \rightarrow+\infty$.

$$
\ln \tilde{A}(t) \sim \ln \left\{\tilde{F}\left(t\left(f\left(x_{+}\right)-f\left(x_{-}\right)\right)\right)+\tilde{F}\left(t\left(f\left(x_{-}\right)-f\left(x_{+}\right)\right)\right\}\right.
$$




\section{Proof.}

Upper Bound. By Proposition 2.5 we get

$$
\begin{aligned}
\tilde{A}(t) & \leqq \int_{\mathbb{R}^{d}} p(t, x)^{2} \int_{C_{0}} d u M\left(e^{-t(q(u)-q(x+u)}\right) \\
& \leqq \sum_{i \in \mathbf{Z}^{d}} \tilde{p}(t, i)^{2} \int_{C_{0}} d u \int_{C_{0}} d w M\left(e^{-t(q(u)-q(i+w)}\right),
\end{aligned}
$$

where $\tilde{p}(t, i):=\sup _{v \in 2 C_{0}} p(t, i+v)$

$$
\begin{aligned}
& \leqq \tilde{p}(t, 0)^{2} \int_{C_{0}} d u \int_{C_{0}} d w M\left(e^{-t \xi_{0}(f(u)-f(w))}\right) \\
& \quad+\left(\sum_{i \in \mathbb{Z}^{d}} \tilde{p}(t, i)^{2}\right) \int_{C_{0}} d u M\left(e^{-t \xi_{0} f(u)}\right) \cdot \int_{C_{0}} d w M\left(e^{+t \xi_{0} f(w)}\right) .
\end{aligned}
$$

First, we estimate the first term above:

$$
\begin{aligned}
\int_{C_{0}} d u \int_{C_{0}} d w M\left(e^{-t \xi_{0}(f(u)-f(w))}\right) \leqq & M_{+}\left(e^{-t \xi_{0}\left(f\left(x_{-}\right)-f\left(x_{+}\right)\right)}\right) \\
& +M_{-}\left(e^{-t \xi_{0}\left(f\left(x_{+}\right)-f\left(x_{-}\right)\right)}\right) .
\end{aligned}
$$

The second term was essentially already treated in our estimate on $\tilde{N}(t)$. It is obvious that the estimate for the first term is dominant.

Lower Bound. We use similar techniques as in Sect. 3. Set $\Omega_{1}=\{x(\cdot)|| x(s) \mid \leqq \eta$ for $0 \leqq s \leqq t \leqq t-\delta\}$. We have

$$
\tilde{A}(t) \leqq C p(t, 0) \int_{C_{0}} d u \int_{C_{0}} d w E_{0,0}^{t, w-u} E_{0,0}^{t, w-u} M\left(e^{-\int_{0}^{t}\left\{q(u+x(s))-q\left(w-x^{\prime}(s)\right)\right\} d s}\right) .
$$

To shorten notation we introduce

then

$$
\mathscr{E}(\cdot)=\int_{C_{0}} d u \int_{C_{0}} d w \int_{\Omega_{1}} d P_{0,0}^{t, w-u} \int_{\Omega_{1}} d P_{0,0}^{t, w-u} \int d P(\cdot)
$$

$$
\tilde{A}(t) \leqq C p(t, 0) \mathscr{E}\left(e^{-\int_{0}^{t}\left\{q(u+x(s))-q\left(w-x^{\prime}(s)\right)\right\} d s}\right)
$$

Now

$$
\begin{aligned}
\mathscr{E}\left(e^{-\int_{0}^{t}\left\{q(u+x(s))-q\left(w-x^{\prime}(s)\right)\right\} d s}\right)= & \mathscr{E}\left(e^{-\int_{0}^{t-\delta}\left\{q(u+x(s))-q\left(w-x^{\prime}(s)\right)\right\} d s}-e^{-\int_{t-\delta}^{t}\{\ldots\} d s}\right) \\
\geqq & \mathscr{E}\left(e^{-\frac{1}{p} t \int_{0}^{t \delta}\left\{q(u+x(s))-q\left(w-x^{\prime}(s)\right)\right\} d s}\right)^{p} \\
& \cdot \mathscr{E}\left(e^{-\frac{q}{p} t \int_{0}^{\delta}\left\{q(u+x(s))-q\left(w-x^{\prime}(s)\right)\right\} d s}\right)^{p / q}
\end{aligned}
$$
by Hölder's inequality for any $p, q$ with $1 \leqq p<\infty$ and $\frac{1}{p}+\frac{1}{q}=1$. We will choose
$p$ later, in fact, we are going to send it to 1 . 
Let us estimate the first factor in (4.7):

$$
\begin{aligned}
& \mathscr{E}\left(e^{-\frac{1}{p} \int_{0}^{t-\delta}\left\{q(u+x(s))-q\left(w-x^{\prime}(s)\right)\right\} d s}\right)=\int_{C_{0}} d u \int_{C_{0}} d w \int_{\Omega_{1}} d P_{0,0}^{t, w-u} \int_{\Omega_{2}} d P_{0, w-u}^{t, 0} \\
& M_{+}\left(e^{-\frac{1}{p} \xi_{0}{ }^{t-\delta}\left\{f(u+x(s))-f\left(w-x^{\prime}(s)\right)\right\} d s}\right)+M_{-}\left(e^{-\frac{1}{p} \xi_{0} \int_{0}^{t-\delta}\left\{f(u+x(s))-f\left(w-x^{\prime}(s)\right)\right\} d s}\right) .
\end{aligned}
$$

Again, by taking $u$ small enough we obtain

so

$$
|f(u+x(s))-f(u)|<\varepsilon \text { for } x() \in \Omega_{1} \quad \text { and } s<t<-\delta,
$$

$$
\begin{aligned}
& \geqq \inf _{y \in C_{0}} P_{0,0}^{t, y}\left(\Omega_{1}\right)^{2}\left\{M_{+}\left(\int_{C_{0}} d u \int_{C_{0}} d w e^{-\frac{t-\delta}{p} \xi_{0}(f(u)-f(w)+2 \varepsilon)}\right)\right. \\
& \left.+M_{-}\left(\int_{C_{0}} d u \int_{C_{0}} d w e^{-\frac{t-\delta}{p} \xi_{0}(f(u)-f(w)+2 \varepsilon)}\right)\right\} \\
& \leqq C e^{-C_{2} \frac{1}{\eta^{2}}} \cdot t^{-t / 2}\left\{M_{+}\left(e^{-\frac{t-\delta}{p} \xi_{0}\left(f\left(x_{-}\right)-f\left(x_{+}\right)+2 \varepsilon\right)}\right)\right. \\
& \left.+M_{-}\left(e^{-\frac{t-\delta}{p} \xi_{0}\left(f\left(x_{+}\right)-f\left(x_{-}\right)-2 \varepsilon\right)}\right)\right\} .
\end{aligned}
$$

Therefore

$$
\lim _{t \rightarrow \infty} \frac{\ln \tilde{A}(t)}{\ln \left\{\tilde{F}\left(t\left(f\left(x_{+}\right)-f\left(x_{-}\right)\right)\right)+\widetilde{F}\left(t\left(f\left(x_{-}\right)-f\left(x_{+}\right)\right)\right)\right\}}=1 .
$$

\section{Discussion}

1. We see that in all considered cases the asymptotics of the ILAC even on the logarithmic scale is more complicated and sensitive to the structure of the random potential than the asymptotics of the IDS. In general the ILAC decreases more slowly than the IDS and thus the former decrease cannot be reduced to the latter one. Such reduction is possible only in the simplest cases of the Gaussian potential with a nonnegative correlation function, when according to Theorem (3.1) we obtain (1.5), or the Poisson (3.2) or alloy-type (4.1) potentials with a constant sign function $f(x)$. It turns out that the case when the asymptotic behaviour of $\tilde{A}(t)$ can be reduced to the asymptotic behaviour of $\tilde{N}^{ \pm}(t)$ can be written in the following transparent asymptotic relation:

$$
\tilde{A}(t)=\tilde{N}^{+}(t) \cdot \tilde{N}^{-}(t), \quad t \rightarrow \infty
$$

(on a logarithmic scale). If e.g. $\tilde{N}^{+}(t)$ dominates $\tilde{N}^{-}(t)$ (as in the mentioned case of the constant sign Poisson or alloy-type potential), when $\ln \tilde{N}^{-}(t)$ $=-$ const $\cdot t^{a}(1+o(1)), t \rightarrow+\infty$ [3]) then the leading behaviour of $\tilde{A}(t)$ is given by $\tilde{N}(t)$. Only in the case when $\tilde{N}^{-}(t)$ and $\tilde{N}^{+}(t)$ behave similarly the leading term of $\tilde{A}(t)$ detects the product $\tilde{N}^{-} \tilde{N}^{+}$. In particular, if the random potential has a symmetric distribution like the Gaussian potential of (4.1) with symmetric $\xi_{i}$ 's we obtain $\tilde{A}(t) \sim\left(N^{+}(t)\right)^{2}$, the squaring behaviour. 
Thus in these cases we have a kind of asymptotic factorisation of $\tilde{A}(t)$. Since, according to (1.1)

and

$$
\tilde{A}(t)=C_{1} \sum_{n, m} e^{-t \lambda_{n}^{-}} e^{-t \lambda_{m}^{+}}\left|\left(\phi_{n}^{-}, \phi_{m}^{+}\right)\right|^{2}
$$

$$
\tilde{N}^{+}(t) \cdot \tilde{N}^{-}(t)=C_{2} \sum e^{-t \lambda_{n}^{-}} e^{-t \lambda_{m}+}
$$

the validity of this asymptotic factorisation can be interpretet as the fact that the quantum mechanical transition probability (rate of tunneling) $\left|\left(\phi_{n}^{-}, \phi_{m}^{+}\right)\right|^{2}$ assumes more or less the same and not too small value for all the states whose energies lie near the spectrum edges $\pm \infty$ of the operator $H^{ \pm}$.

2. Our results have an additional aspect. Namely, let us consider the timedependent potential

$$
q_{t}(s, x)=\left\{\begin{aligned}
q(x), & 0 \leqq s<t \\
-q(x), & t \leqq s \leqq 2 t
\end{aligned}\right.
$$

Then our basic representation of $\tilde{A}(t)$ from Corollary 2.1 can be written as

$$
\tilde{A}(t)=M\left(p(2 t, 0) E_{0,0}^{0,2 t}\left(e^{-\int_{0}^{2 t} q_{t}(s, x(s)) d s}\right)\right),
$$

since, by the Feynman-Kac formula, the expression under $M(\ldots)$ sign is the fundamental potential (5.2). Our asymptotic formulas from Proposition 3.3, 3.4, and 4.2 show that for a time-dependent random potential varying on the scale $t$ the mathematical expectation of the fundamental solution of the respective parabolic equation may differ for large time from the corresponding solution for the timeindependent random potentials.

3. In this paper we considered random potentials that have so-called [3] classical asymptotics of the IDS, i.e. asymptotics whose leading terms are determined solely by the random potential and do not contain the Planck constant or the mass of the particle (constant in front of the Laplacian). The more complicated cases arise when the kinetic and potential energy terms of the Schrödinger operator give the same order contributions to the respective asymptotic formulas. These asymptotic formulas are all called the quantum case. They arise if the edges $\lambda_{g}^{ \pm}$of the spectrum of $H^{ \pm}$either are not $\pm \infty$ or if $\lambda_{g}= \pm \infty$, but the respective random potentials are singular (Gaussian white noise, Poisoon or alloy-type potential with point single impurity potential $f(x)$ or with screened Coulomb potential $f(x)=-$ const $\left.\frac{e^{|x| / r_{0}}}{|x|}\right)$. We will consider respective asymptotics for the ILAC elsewhere. Here we only mention a general lower bound that replaces the simple Wiener integral argument which we used in Sect. 3 and 4. As it is shown in [3], the variational lower bound for $\tilde{N}(t)$ that asymptotically coincides with all known exact asymptotic formulas for $\tilde{N}(t)$ (both in classical and quantum cases)

$$
\tilde{N}(t) \geqq\|\psi\|_{1}^{-2} M\left(\exp \left\{-t \int|\nabla \psi|^{2} d x-t \int\left(q(x) \psi^{2}(x) d x\right)\right\}\right),
$$

where the "trial" function $\psi(x)$ is nonnegative, belongs to $L^{2}\left(\mathbb{R}^{d}\right) \cap C^{1}\left(\mathbb{R}^{d}\right)$ and $\|\psi\|_{2}=1$. In the classical cases (Gaussian, Poisson, or alloy-type with continuous 
$B(x)$ and $f(x)$ respectively), taking $\psi(x)=R^{-d / 2} \varphi\left(\frac{x}{R}\right)$, where $\|\varphi\|_{L^{2}}=1$ and $R \rightarrow \infty$ as $t \rightarrow \infty$, we find that the kinetic energy term $t \int|\nabla \psi|^{2} d x$ is negligible in comparison with the potential energy $\ln M\left(\exp \left\{-\left.t \int \nabla \psi\right|^{2} d x\right\}\right)$. It turns out that there exists an analog of the variational bound (5.3) for the ILAC. Since, as we have seen, the Laplace transform of the ILAC can be written as the mathematical expectation of the diagonal element of the fundamental solution of the parabolic equation with the time-dependent random potential (5.2), we will formulate the above analog in this more general setting. Let $q(t, x)$ be a time-dependent potential and $H(t)=-\Delta$ $+q(t)$ be the Schrödinger operator with this potential. Denote by $P\left(t_{0}, t\right)$ the solution of the operator-valued Cauchy problem $\frac{\partial P}{\partial t}=P H(t), P\left(t_{0}, t_{0}\right)=1$ (it can be represented either as some Wiener integral or as a time-ordered operator exponent). Consider also a "trial" time dependent potential $v(t, x)$ and the respective Cauchy solution $Q\left(t_{0}, t\right)$. Then for any nonnegative $\psi \in L^{2}\left(\mathbb{R}^{d}\right)$,

$$
(P(0,2 t) \psi, \psi) \geqq Z_{t} \exp \left\{-\int_{0}^{2 t}(Q(0, s)(q(s)-v(s)) Q(s, 2 t) \psi \psi)\right\},
$$

where $Z_{t}=(\psi, Q(0,2 t) \psi)$.

It can be shown that for the potential (5.2) it suffices to take a time piece-wise constant potential $v(s, x)$ :

$$
v_{t}(s, x)= \begin{cases}v_{1}(x), & 0 \leqq s<t \\ v_{2}(x), & t \leqq s<2 t\end{cases}
$$

where $v_{1,2}(x)$ are nonrandom, bounded from below and tending to $+\infty$ as $|x| \rightarrow \infty$ potentials and $\psi$ is the ground state of $-\Delta+v_{1}$. The variational bound (5.3) can be obtained by using the Jensen inequality in the Wiener integral representation of lefthand side of (5.3). The proof of inequality (5.3) and its applications will be published elsewhere.

\section{Appendix}

In the appendix we sketch a proof that the ILAC $A(\lambda)$ is independent of the boundary conditions chosen to define it. This proof is close to the analogous procedure for the density of states (see [8] or [10]).

Let us indicate the Dirichlet and Neumann Hamiltonians by a superscript $D$ and $N$ respectively. We set

$$
\tilde{A}_{\Lambda}^{N}(t)=\frac{1}{V} \operatorname{tr} e^{-t H_{A}^{+}, N} e^{-t H_{A}^{-}, N}
$$

and define $\tilde{A}_{A}^{D}$ analogously.

It is known that the integral kernel of $e^{-t H^{-}, N}$ has a representation in terms of Brownian motion. To make this precise, let us introduce a function $\varphi: \mathbb{R}^{d} \rightarrow[-L / 2, L / 2]^{d}$ by:

$$
\varphi_{i}(x)=\left\{\begin{array}{ll}
x_{i}-2 n L & \text { if }(2 n-1 / 2) L \leqq x_{i} \leqq(2 n+1 / 2) L \\
(2 n+1) L-x_{i} & \text { if }(2 n+1 / 2) L \leqq x_{i} \leqq(2 n+3 / 2) L
\end{array} \text { for } n \in \mathbb{Z},\right.
$$


then the integral kernel of $e^{-t H_{A}^{+} N}$ is given by:

(see [12, 6.3.11 and 6.3.12]).

$$
e^{-H_{A}^{+}, N}(x, y)=\sum_{z \in \varphi^{-1}(y)} E_{0, x}^{t, z}\left(e^{-\int_{0}^{t} q(\varphi(x(s)) d s}\right)
$$

Therefore we conclude

$$
\begin{gathered}
0 \leqq \\
\tilde{A}_{\Lambda}^{N}(t)-\tilde{A}_{\Lambda}^{D}(t)=\frac{1}{V} \int_{\Lambda} d x \int_{\Lambda} d y \sum_{z, z^{\prime} \in \varphi^{-1}(y)} E_{0, x}^{t, z} E_{0, x}^{t, z^{\prime}} \\
\left(e^{-\int_{0}^{t} q(\varphi(x(s)))-q\left(z^{\prime}-\varphi(y(s))\right) d s} \tilde{\chi}_{\Lambda}(x(s)) \tilde{\chi}_{\Lambda}(y(s))\right), \\
\text { here } \tilde{\chi}_{\Lambda}(x(s))= \begin{cases}1 & \text { if } x(s) \notin \Lambda \text { for some } 0 \leqq s \leqq t \\
0 & \text { otherwise }\end{cases}
\end{gathered}
$$

By the Cauchy-Schwarz inequality this expression can be estimated by

$$
\begin{aligned}
& \left(\frac{1}{V} \int_{\Lambda} d x \int_{\Lambda} d y \sum_{z, z^{\prime} \in \varphi^{-1}(y)} E_{0, x}^{t, z} E_{0, x}^{t, z^{\prime}}\left(e^{-2 \int_{0}^{t} q(\varphi(x(s)))-q\left(z^{\prime}-\varphi(y(s))\right) d s}\right)\right)^{1 / 2} \\
& \left(\frac{1}{V} \int_{\Lambda} d x \int_{\Lambda} d y \sum_{z, z^{\prime} \in \varphi^{-1}(y)} E_{0, x}^{t, z} E_{0, x}^{t, z^{\prime}}\left(\tilde{\chi}_{\Lambda}(x(\cdot)) \tilde{\chi}_{\Lambda}(y(\cdot))\right)\right)^{1 / 2} .
\end{aligned}
$$

The first factor can be estimated in terms of the prelimit densities of states $N_{A}^{ \pm}, N$, (in analogy to (2.8)) and hence is bounded. The second factor is just $\tilde{A}_{\Lambda}^{N}-\tilde{A}_{\Lambda}^{D}$ for $q=0$, thus this term can be esitmated by

$$
\frac{1}{V} \operatorname{tr}\left(e^{-2 t H_{0_{\Lambda}}^{N}}-e^{-2 t H_{0_{\Lambda}}^{D}}\right)
$$

which is well known to converge to zero (see $[8,10])$.

Acknowledgements. We are grateful to S. R. S. Varadan for the stimulating discussion of the meaning of formula (5.3). L. A. Pastur would like to thank S. Albeverio for the kind invitation to the BiBoS research center Bielefeld and the SFB 237 at the Ruhr-Universität Bochum. W. Kirsch thanks the Institute for Low Temperature Physics and Engeneering of the Ukrainian Academy of Sciences for its hospitality. Financial support through these institutions is gratefully acknowledged.

\section{References}

1. Bonch-Bruevich, V.L., Enderlein, R., Esser, B., Kneiper, R., Mironov, A.G., Zuyagagin, I.P.: Elektronentheorie Ungeordneter Halbleiter. Berlin: Deutscher Verlag der Wissenschaften 1984

2. Efros, A.L., Shklowski, B.L.: Electronic properties of doped semiconductors. Berlin Heidelberg New York: Springer 1984

3. Lifshitz, I.M., Gredeskul, S.A., Pastur, L.A.: Introduction in the theory of disordered systems. Moskau: Nauka 1982 and New York: Wiley 1988

4. Arbuzov, Yu.D., Evdokimov, V.M., Kolenkin, M.Yu.: JETP 92, 1351-1356 (1987) 
5. Reed, M., Simon, B.: Methods of modern mathematical physics. IV. Analysis of operators. New York: Academic Press 1978

6. Simon, B.: Functional integration and quantum physics. New York: Academic Press 1979

7. Krengel, U.: Ergodic theorems. Betlin: de Gruyter 1985

8. Pastur, L.A.: Russ. Math. Surveys 28, 1-67 (1973)

9. Gikhman, I.I., Skorokhod, A.V.: Introduction to the theory of random processes. Philadelphia: Saunders 1969

10. Kirsch, W., Martinelli, F.: J. Phys. A 15, 2139-2156 (1982)

11. Kirsch, W.: Adv. Appl. Math. 6, 177-187 (1985)

12. Bratelli, O.: Robinson, D.W.: Operator algebras and quantum statistical mechanics. II. Berlin Heidelberg New York: Springer 1981

Communicated by T. Spencer

Received May 25, 1989; in revised form March 9, 1990 